\title{
Case Report on Phenytoin Induced Cerebellar Syndrome
}

\author{
Gautam Satheesh ${ }^{1 *}$, Sujith Varma $K^{2}$, Anil Antony ${ }^{3}$ \\ ${ }^{1}$ Department of Pharmacy Practice, National College of Pharmacy, Calicut, Kerala, INDIA. \\ ${ }^{2}$ Associate Professor and Head, Department of Pharmaceutics, National College of Pharmacy, Calicut, Kerala, INDIA. \\ ${ }^{3}$ Department of Pharmacy Practice, National College of Pharmacy, Calicut, Kerala, INDIA.
}

\begin{abstract}
Phenytoin, an anticonvulsant extensively used to control generalized tonic-clonic seizures as well as simple and complex partial seizures, has a stabilizing influence on neuronal membrane. Phenytoin acts by prolonging the inactivated state of sodium channel, thereby extending the refractory period of neurons and limiting the repetitive firing of action potentials. Bioavailability of phenytoin fluctuates widely on the account of pharmacokinetic variability. Consequently, the narrow therapeutic window of phenytoin frequently causes adverse effects and toxicity. Phenytoin at higher doses is associated with various vestibular and cerebellar side effects like motor ataxia, muscle spasms, psychoses and visual disturbances. Long term use of phenytoin at therapeutic and toxic levels can lead to cerebellar atrophy. However, phenytoin-induced cerebellar syndrome is reversible on timely withdrawal of the medication. Regular monitoring of plasma concentrations, accurate dosing, and medication adherence to treatment regimens are important. Here, we report a case of phenytoin-induced cerebellar syndrome presenting with behavioral abnormalities, paraparesis and visual impairment. The patient's condition improved upon gradually tapering the dose, followed by termination of phenytoin therapy and substitution with carbamazepine. This report emphasizes on the importance of regular monitoring of plasma drug concentration, accurate dosing of drugs having a narrow therapeutic index, and identification of noncompliance in patients treated with phenytoin.
\end{abstract}

Key words: Phenytoin, Cerebellar syndrome, Epilepsy, Adverse drug reaction.

\section{INTRODUCTION}

Phenytoin is widely used for controlling generalized and partial seizures due to its stabilizing influence on neuronal membrane without causing general depression of the central nervous system. Phenytoin acts by prolonging the inactivated state of voltage activated sodium channels and governs the refractory period of neurons, thus limiting the repetitive firing of action potentials. Five to tenfold higher doses of phenytoin can reduce the calcium influx, inhibit glutamate and facilitate $\gamma$-aminobutyric acid (GABA) responses. These effects may also underlie some of the inappropriate toxicity associated with the higher serum levels of phenytoin. ${ }^{1}$
Three factors govern the pharmacokinetic features of phenytoin; the extent of protein binding, non-linear elimination kinetics and its ultimate metabolism by the liver. Up to $90 \%$ of the administered phenytoin is protein bound, and there exists a distinction between the total and free plasma levels of phenytoin. Even small variations in protein binding can drastically alter the amount of free drug in the serum. Phenytoin is metabolized mostly in the liver by cytochrome P450 isoforms CYP2C9/10 and CYP2C19. ${ }^{1}$

The pharmacokinetic variability and narrow therapeutic index of Phenytoin often lead to toxicity. The usage of higher doses
DOI: 10.5530/ijopp.11.1.11

Address for correspondence: Gautam Satheesh, Department of Pharmacy Practice, National College of Pharmacy, Manassery, Calicut, Kerala-673602, INDIA. Phone no: +918848494964 Email Id: gautamsatheesh@ gmail.com

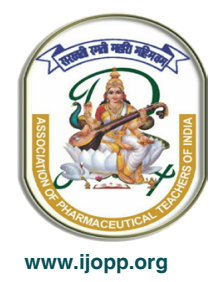


of phenytoin for longer duration is associated with cerebellar and vestibular toxicity which manifest as sedation, visual disturbances, ataxia, nystagmus, muscle spasms, and psychosis. Other potential toxic effects of phenytoin include cardiac arrhythmias, hypotension, megaloblastic anaemia, osteomalacia, gingival hyperplasia, hepatotoxicity and hypersensitivity reactions. ${ }^{1}$ The longterm use of phenytoin at therapeutic and toxic levels can cause cerebellar changes including atrophy. However, the phenytoin induced cerebellar syndrome can be reversed by timely cessation of phenytoin therapy. Regular monitoring of plasma concentration, accurate dosing, and compliance to treatment regimens are important to obtain the best treatment outcome.

\section{CASE REPORT}

A female patient aged 36 years experiencing simple partial seizures following a meningioma surgery was on phenytoin therapy for four months. The patient's major complaints were diminished vision, mild behavioural abnormalities, slurred speech, difficulty in walking, and multiple recent episodes of fall. The patient had an episode of simple partial seizure 2 days prior to her hospitalization. The patient underwent a craniotomy four month before her hospitalization, for the removal of a meningioma which manifested as oedema and compression in the brain parenchyma. The patient's family history was insignificant with respect to her condition.

The patient was conscious and oriented at the time of hospital admission. On neurological examination, the patient's abnormal gait was evaluated. The routine laboratory examinations could not reveal any explanatory reasons for the present condition of the patient. The liver and renal function tests were also found to be within normal limits.

The patient's behavioral abnormalities started to worsen on the third day of admission. The MRI scan of the brain showed mild cerebellar atrophy, which may be due to the high doses of phenytoin. The MRI scan of the brain and the spinal cord however revealed no residual compression or edema. The patient was referred to a psychiatrist who suggested the possibility of phenytoin toxicity. The patient's serum phenytoin level was found to be $41.6 \mu \mathrm{g} / \mathrm{ml}$, which lies outside the therapeutic range of phenytoin $(10-20 \mu \mathrm{g} / \mathrm{ml})$. The patient was advised to take carbamazepine following the initial tapering of doses and ultimate cessation of phenytoin therapy. A similar diagnosis was made by the ophthalmologist following the consultation for the complaints of diminished vision.
Patient's phenytoin dose was tapered from $300 \mathrm{mg} /$ day and gradually stopped. Patient was simultaneously treated with Carbamazepine (200mg/day), Clonazepam $(0.25 \mathrm{mg}$ / day) and Clobazam $(10 \mathrm{mg} /$ day). The condition of the patient improved symptomatically and was discharged after five days. The patient was asked to consult the neurologist a week after discharge from the hospital. The patient is now stable and did not experience any episodes of seizure or abnormal gait after the discharge.

\section{DISCUSSION}

Phenytoin, being a popular anticonvulsant widely used for both generalized and partial seizures, is generally considered safe. The pharmacokinetics of phenytoin follow a non-linear path i.e. the rate of elimination varies as a function of concentration. At very low plasma levels, the elimination follows first order kinetics. However, in the therapeutic range, only a small proportion of the drug is metabolized because of saturation of enzymatic pathways. Above the therapeutic range, even a small increase in the dose can markedly elevate the plasma concentration as well as the half-life of phenytoin. This shift from first order to zero order kinetics occurs unpredictably. ${ }^{1}$ At plasma concentrations of $10 \mu \mathrm{g} / \mathrm{ml}$, the plasma half-life of phenytoin ranges between 6 to $24 \mathrm{~h}$, which however may vary with higher concentrations. ${ }^{1}$ Normally, approximately $90 \%$ of the circulating phenytoin is bound to albumin, whereas the therapeutic free phenytoin levels are 1-2 $\mu \mathrm{g} / \mathrm{ml}$. The inappropriate absorption of phenytoin into cells which leads to undesirable side effects, is largely from the free plasma pool. Individuals with decreased protein binding may show clinical toxicity even at normal level total phenytoin in blood plasma. However, such individuals have an elevated free phenytoin level. Thus, the measurement of free drug concentration in the serum can be a useful aid in the assessment of phenytoin toxicity. ${ }^{1}$

Phenytoin has a potential to cause various adverse effects, at both therapeutic and toxic doses. Many dosebased adverse drug reactions have been reported for phenytoin, among which sedation, gum hypertrophy and hirsutism are the most common ADRs at therapeutic doses. Cerebellar and vestibular toxicity, which manifest as dizziness, ataxia and nystagmus, have been reported at therapeutic doses as well as when the serum drug levels are above the therapeutic range. ${ }^{2,3}$

The plasma drug concentration rises disproportionately, when the dose of phenytoin is increased. Thus, the plasma concentration of phenytoin can rise from sub therapeutic level to toxic levels even with small increase 
in dose. Therefore, accurate dosing and maintenance of correct levels of phenytoin in serum are of prime importance to prevent toxicity. Phenytoin toxicity may be severe with associated co-morbidities like renal and hepatic diseases, hypothyroidism, hypoalbuminemia and Acquired Immuno Deficiency Syndrome (AIDS). ${ }^{3}$ Earlier studies have reported the association of phenytoin use with transient chorea, athetosis, myoclonus, orofacial abnormality and dystonia. ${ }^{4}$ Other marked symptoms like abnormal gait and ataxia are often accompanied by nystagmus, dysarthria (slurring of speech), suggestive of phenytoin's adverse effect on the cerebellum. ${ }^{5}$ Various cases of cerebellar atrophy have been reported on longterm use of phenytoin, even at therapeutic doses. ${ }^{6}$

In the present case study, the patient showed symptoms of mild behavioural changes which started to worsen over time due to high dose of phenytoin. The serum phenytoin concentration of the patient was $41.6 \mu \mathrm{g} / \mathrm{ml}$. These transient neurotoxic and ophthalmic side effects have been reported to occur during first few hour of drug intake. These side effects may be due to the excessive fluctuation in plasma concentration of phenytoin between drug administration and the time taken to achieve peak plasma concentration. ${ }^{7}$ There is no definite correlation of serum levels of phenytoin with neurological features of toxicity. Toxic adverse effects of phenytoin may also develop at therapeutic doses. The correlation between serum level of anti-epileptic drugs, and their side effects has always been unpredictable. ${ }^{7}$ The casualty assessment of the adverse event of the patient in the present study scored 8 on the Naranjo scale, which indicates a probable adverse drug reaction.

\section{CONCLUSION}

Phenytoin is generally considered safe for use to control seizures and is extensively used in the treatment of all types of partial and Grand mal seizures. However, the patient in this case study started recovering steadily after the drug was withdrawn. Hence, regular monitoring of adverse drug reaction should be considered in patients undergoing phenytoin therapy on long term basis. These adverse effects are acute or chronic in nature and can also be idiosyncratic or dose-dependent. As most of these effects are reversible on cessation of therapy, early identification and appropriate management of adverse

\begin{tabular}{|c|c|}
\hline $\begin{array}{c}\text { Serum Phenytoin level } \\
(\mu \mathrm{g} / \mathrm{ml})\end{array}$ & Corresponding symptoms \\
\hline$<10 \mu \mathrm{g} / \mathrm{ml}$ & Rare \\
\hline $10-20 \mu \mathrm{g} / \mathrm{ml}$ & Occasional mild nystagmus \\
\hline $20-30 \mu \mathrm{g} / \mathrm{ml}$ & Nystagmus \\
\hline $30-40 \mu \mathrm{g} / \mathrm{ml}$ & Ataxia, slurred speech, vomiting \\
\hline $40-50 \mu \mathrm{g} / \mathrm{ml}$ & Confusion, delirium, lethargy \\
\hline$>50 \mu \mathrm{g} / \mathrm{ml}$ & Seizures, coma \\
\hline
\end{tabular}

drug reactions are of prime importance. The present study also emphasizes on the importance of regular monitoring of plasma drug concentration, accurate dosing of drugs having a narrow therapeutic index, and identification of noncompliance in patients treated with phenytoin.

\section{CONFLICTS OF INTEREST}

The authors have declared no conflicts of interests.

\section{ACKNOWLEDGEMENT}

The authors thank the Department of Neurology, KMCT Medical College Hospital, for their valuable support throughout the study.

\section{REFERENCES}

1. Hardman JG, Limbird LE, Molinoff PB, Ruddon RW. Goodman and Gilman's The Pharmacological Basis of Therapeutics. 9th Ed. New York: McGrawHill. 1996;461-86.

2. Kuruvilla T, Bharucha NE. Cerebellar atrophy after acute phenytoin intoxication. Epilepsia. 1997;38(4):500-2.

3. McNamara JO, Hwang WJ, Tsai JJ. Acute phenytoin intoxication: Causes, symptoms, misdiagnoses, and outcomes. Kaohsiung $\mathrm{J}$ Med Sci. 2004;20(12):580-5.

4. Chadwick D, Reynolds EH, Marsden CD. Anticonvulsant-induced dyskinesias: A comparison with dyskinesias induced by neuroleptics. J Neurol Neurosurg Psychiatry. 1976;39(12):1210-8.

5. Brostoff JM, Birns J, McCrea D. Phenytoin toxicity: an easily missed cause of cerebellar syndrome. Journal of Clinical Pharmacy and Therapeutics. 2008;33:211-4.

6. McLain LW, Martin JT, Allen JH. Cerebellar degeneration due to chronic phenytoin therapy. Ann Neurol. 1980;7(1):18-23.

7. Lüders $\mathrm{H}$, Acharya J, Baumgartner $\mathrm{C}$, Benbadis S, Bleasel A, Burgess R, et al. Semiological seizure classification. Epilepsia. 1998;39(9):1006-13. 